\title{
Closure of pancreatic intraductal papillary mucinous carcinoma-colonic fistula using an over-the-scope clip
}
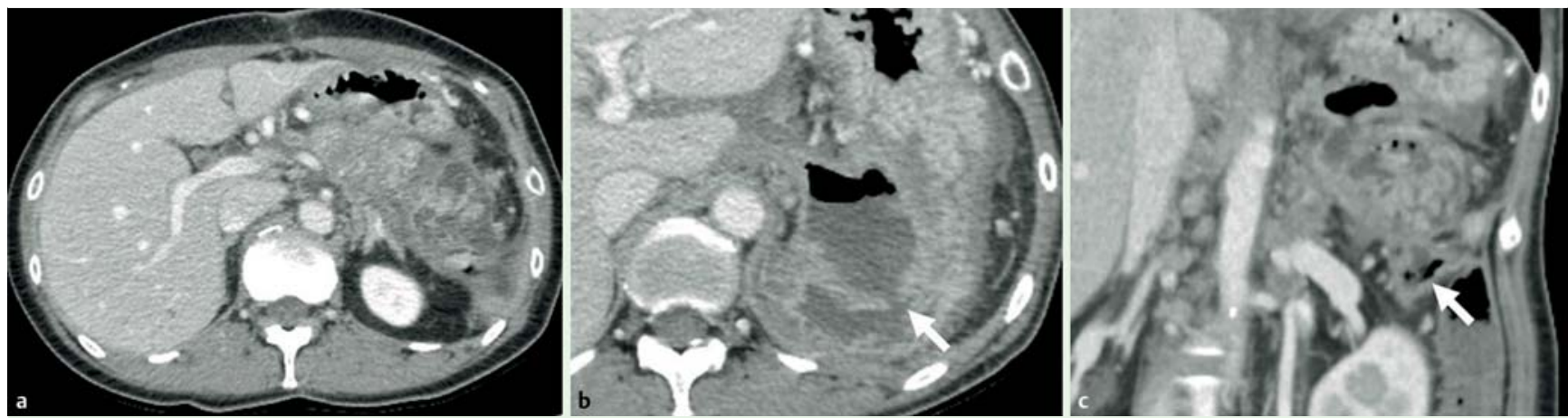

Fig. 1 Contrast-enhanced computed tomographic images. a A poorly contrast-enhanced mass, $40 \mathrm{~mm}$ in diameter, was seen in the body of the pancreas. A cystic lesion was found on the caudal side of the pancreas, and contrast-enhanced nodules were seen inside the lesion. $\mathbf{b}$ A fistula between the stomach and the pancreatic cyst was suspected (arrow). c A fistula between the transverse colon and the pancreatic cyst was suspected (arrow).
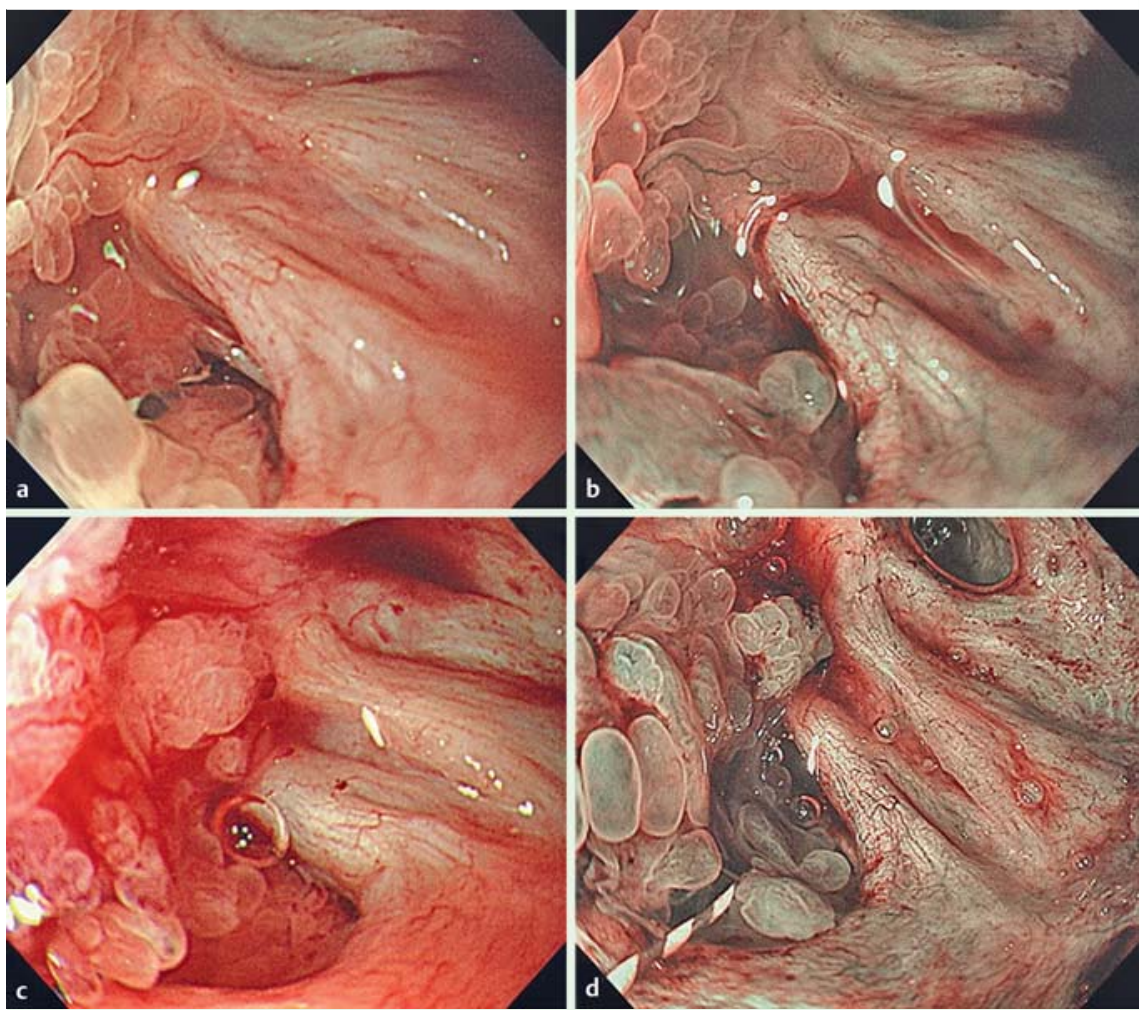

Fig. 2 Gastric fistula associated with intraductal papillary mucinous carcinoma. Endoscopic view showing type 4 papillary nodules (a, b). Endoscopic image showing type 3 papillary nodules (c, $\mathbf{d})$. a, c Conventional endoscopic image; $\mathbf{b}, \mathbf{d}$ narrow-band image.

Pancreatic intraductal papillary mucinous carcinoma (IPMC) can rupture and lead to fistula formation in multiple organs [1,2]. An over-the-scope clip (OTSC) has been used to close gastrointestinal perforations and fistulas [3]. We describe a patient in whom the OTSC was useful for the closure were diagnosed. Even if surgery was performed, curative resection was impossible, and peritoneal drainage and splenectomy were thus performed. The patient was referred to our hospital.

Chemotherapy was begun, but a second rupture occurred 2 months after the initial rupture. The patient's condition responded to medical treatment, and chemotherapy was resumed. A high fever and high levels of inflammatory markers were noted 4 months after the initial rupture. Contrast-enhanced computed tomography suggested fistula formation in the stomach and transverse colon ( $\boldsymbol{\bullet}$ Fig. $\mathbf{1}$ ). Upper endoscopy was performed.

The fistula site was dilated and the endoscope was inserted. Type 3 and 4 papillary nodules [4] were seen ( $\bullet$ Fig. 2 , $\odot$ Fig. 3, - Video 1). Biopsy revealed adenocarcinoma. Colonoscopy showed mucus outflow and type 3 papillary nodules in the splenic flexure. Infectious IPMC caused by fecal influx was highly suspected. The infection improved after fasting and treatment with antibiotics. Then, the OTSC system (Ovesco Endoscopy AG; Tübingen, Germany) was used to close the IPMCcolonic fistula ( $\bullet$ Video 2 ).

Infection did not recur before the patient died of cancer. In addition, there was no recurrence of rupture associated with closure of the IPMC-colonic fistula. Fistula closure with an OTSC can be a treatment option for unresectable and infectious IPMC-colonic fistulas.

Endoscopy_UCTN_Code_TTT_1AR_2AD 

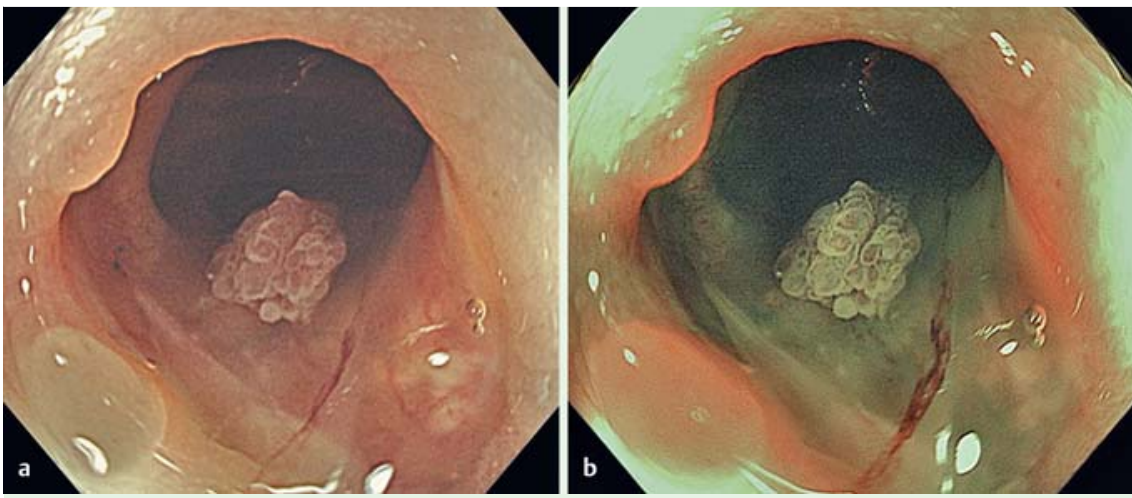

Fig. 3 Colonic fistula associated with intraductal papillary mucinous carcinoma. Endoscopic view showing mucus outflow and type 3 papillary nodules. a Conventional endoscopic image; b narrowband image.

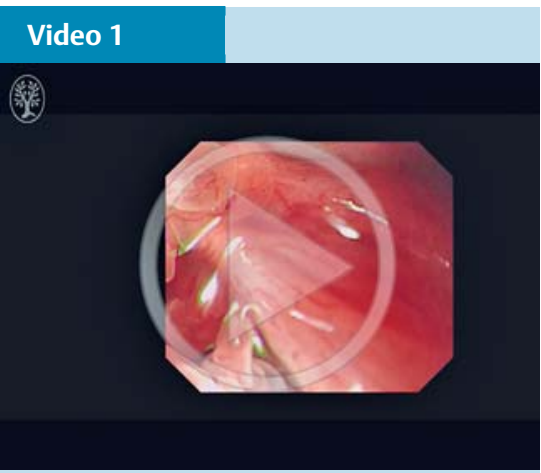

Gastric fistula associated with intraductal papillary mucinous carcinoma. Mucus outflow was present in the posterior wall of the upper gastric body. The fistula site was dilated with a large balloon, and an endoscope was inserted. Endoscopy showed mucous retention on the inside, with evidence of type 3 and 4 papillary nodules. Adenocarcinoma was diagnosed on biopsy.

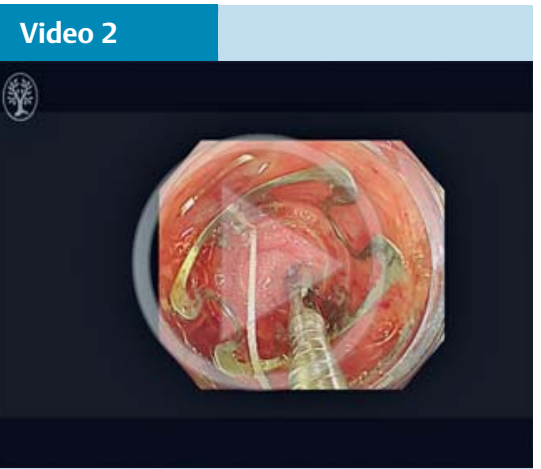

Colonic fistula associated with intraductal papillary mucinous carcinoma (IPMC). Colonoscopy showed extramural compression of the transverse colon and splenic flexure. Mucus outflow was found at the same site. Type 3 papillary protrusions were present inside, and colonic fistula associated with IPMC was diagnosed. The mucosa at the site of fistula was grasped with a forceps, but was torn off because the fistula had formed 4 months earlier and was stiff with adhesions. The fistula could therefore not be pulled into the tip cap. Suction was applied, and the fistula was closed using an over-the-scope clip.
Competing interests: None

\section{Hiroshi Yamauchi, Mitsuhiro Kida, Eiji Miyata, Kosuke Okuwaki, Kana Kawagishi, Hiroshi Imaizumi, Wasaburo Koizumi}

Department of Gastroenterology, Kitasato University School of Medicine, Sagamihara city, Kanagawa, Japan

\section{References}

1 Shimizu M, Kawaguchi A, Nagao S et al. A case of intraductal papillary mucinous neoplasm of the pancreas rupturing both the stomach and duodenum. Gastrointest Endosc 2010; 71: 406-412

2 Lee SE, Jang JY, Yang SH et al. Intraductal papillary mucinous carcinoma with atypical manifestations: report of two cases. World J Gastroenterol 2007; 13: 1622 - 1625

3 Manta R, Manno M, Bertani H et al. Endoscopic treatment of gastrointestinal fistulas using an over-the-scope clip (OTSC) device: case series from a tertiary referral center. Endoscopy 2011; 43: 545-548

4 Hara T, Yamaguchi T, Ishihara T et al. Diagnosis and patient management of intraductal papillary-mucinous tumor of the pancreas by using peroral pancreatoscopy and intraductal ultrasonography. Gastroenterology 2002; 122: 34-43

\section{Bibliography}

Dol http://dx.doi.org/

10.1055/s-0042-117349

Endoscopy 2016; 48: E315-E316

(c) Georg Thieme Verlag KC

Stuttgart · New York

ISSN 0013-726X

\section{Corresponding author}

Hiroshi Yamauchi, MD

Department of Gastroenterology

Kitasato University School of Medicine

1-15-1 Kitasato

Minami-ku, Sagamihara

Kanagawa 252-0375

Japan

Fax: +81-42-7788390

yhiroshi@kitasato-u.ac.jp 